\title{
Positivity conditions on correlation functions that imply Debye screening
}

\author{
Paul Federbush
}

Department of Mathematics, The University of Michigan, Ann Arbor, Michigan 48109

(Received 13 July 1978)

In the classical statistical mechanics setting, a set of positivity conditions on certain twopoint correlation functions is exhibited that implies Debye screening for a large class of Coulomb-like models. For example, for the model treated by Brydges, for which he has rigorously proved shielding, in a range of parameters where $\left\langle\phi^{s}(x) J(y)\right\rangle \geq 0$ for all $x$ and $y$ and all $s$ odd, there is screening. (Alternative conditions require positivity for only two correlation functions.) Strong estimates are obtained for the rate of exponential falloff.

Currently there is much interest in acquiring understanding of Debye screening. An outstanding problem is the question of proving shielding in quantum statistical mechanics (assuming it is valid). Toward this end, the short range difficulties of the $1 / r$ potential have been controlled. ${ }^{1}$

Brydges has recently proved screening for classical Coulomb systems. ${ }^{2}$ We feel the present approach may provide insight into the problem, dealing with shielding charges in a suggestive way. We will deduce exponential falloff of the two point correlation function, for a large class of models, under the assumption of positivity for certain two-point correlation functions. This work may be interesting for a number of reasons. First, it is possible a proof of these positivity conditions will be forthcoming, giving a new proof of shielding. Second, we obtain strong statements on the rate of exponential falloff. Third, the positivity conditions may be tested for in numerical experiments (or theoretically) to provide a good estimate of the range of parameters for which shielding holds.

We study a classical statistical mechanical system of several species of "charged" particles, species $i$ with charge $q_{i}$ and fugacity $z_{i}$. See Ref. 3 , for example, for the basic definitions. The partition function is written as

$$
Z=\sum \prod_{i}\left(\frac{z_{i}^{n_{i}}}{n_{i} !} \int d x^{n_{i}}\right) e^{-\beta V}
$$

with

$$
V=\frac{1}{2} \int: J\left(\frac{\gamma}{r}+v\right) J
$$

$v$ the short range potential, $\gamma / r$ the Coulomb term, and

$$
J=\sum q_{i} \rho_{i}
$$

$\rho_{i}$ the density of species $i$. We assume for convenience

$$
\sum z_{i} q_{i}=0
$$

We define $\phi$ as

$$
\phi=\int\left(\frac{\gamma}{r}+v\right) J
$$

It is helpful to define the notation:

$$
\begin{aligned}
& Z=I\left(e^{-\beta V}\right), \\
& {[A]=I\left(e^{-\beta V} A\right),} \\
& \langle A\rangle=\frac{[A]}{Z},
\end{aligned}
$$

to discuss ensemble averages of a function $A$. We also define

$$
w=(-\Delta) u \text {. }
$$

We are now prepared to state several conditions from which we will prove a number of results.

Condition I: There exists $f(z, x) \equiv f(z)$ (i.e., the $x$ dependence is suppressed) satisfying

$$
\begin{gathered}
\left(-\Delta+4 \pi \gamma \sum_{i} z_{i} q_{i}^{2} \beta+\sum_{i} z_{i} q_{i}^{2} \beta w *\right) f(z) \\
=4 \pi \gamma \delta(z-x)+w(z-x),
\end{gathered}
$$

with $f \geqslant 0$ all $z$ (and $f$ falling off exponentially).

Later we will show such an $f$ exists in a number of interesting cases, and find the falloff explicitly in these cases.

Condition II: $\langle J(y) \phi(x)\rangle \geqslant 0$ all $x, y$,

Condition III.1: $\left\langle J(y) \phi^{s}(x)\right\rangle \geqslant 0$ all $x, y, \quad s$ odd,

Condition III.2:

$\left\langle J(y)\left(-J(x)-\sum_{i} z_{i} q_{i}^{2} \beta \phi(x)\right)\right) \geqslant 0$ all $x \neq y$,

\section{Condition III.3:}

$\left\langle J(y) \sum_{i} z_{i} q_{i}\left(-e^{-\beta q_{i} \phi(x)}+1-\beta q_{\phi} \phi(x)\right)\right\rangle \geqslant 0$ all $x, y$.

A system is charge symmetric if the species occur in pairs with equal fugacities and equal and opposite charges.

Theorem 1: For a system satisfying Conditions I, II, and III.3, or for a system satisfying Conditions I, II, and III.2, or for a charge symmetric system satisfying Conditions I, II, and III.1, one has

$$
0 \leqslant\langle(y) \phi(x)\rangle \leqslant c f(y, x) .
$$


From the conditions on $f$, this implies the exponential falloff of the two point correlation function; the system shields.

For the system studied by Brydges, suitably scaled, (10) becomes

$$
\left(-\Delta+2 z q^{2} \beta\right) f=4 \pi \delta,
$$

an equation on a unit lattice, $\Delta$ the discrete Laplacian. [we have picked his $l=1$, and $\gamma=(1 / 4 \pi)$.]

Theorem 2: For this system one may pick

$$
0 \leqslant f(x, y)=g(x-y)=g\left(x_{1}, x_{2}, x_{3}\right),
$$

where $g$ is the fundamental solution of (16), some of whose properties are given in the Appendix.

We note that it is not known that Conditions II or III hold in the region where Brydges has proved shielding, but we believe they do.

We next consider a system with

$$
v=-\gamma \frac{e^{-\alpha r}}{r} \text {. }
$$

This choice eliminates the singularity of the total potential at $r=0$. Equation (10) in momentum space becomes

$$
\left(k^{2}+\frac{4 \pi \alpha^{2} \tilde{\gamma}}{k^{2}+\alpha^{2}}\right) \tilde{f}=\alpha^{2} \tilde{\gamma} \frac{4 \pi}{k^{2}+\alpha^{2}},
$$

with

$$
\tilde{\gamma}=\sum z_{i} q_{i}^{2} \beta \gamma
$$

We pick

$$
\begin{aligned}
& \tilde{f}=\frac{4 \pi \alpha^{2} \tilde{\gamma}}{r_{2}-r_{1}}\left(\frac{1}{k^{2}+r_{1}}-\frac{1}{k^{2}+r_{2}}\right), \\
& r_{2}=\frac{\alpha^{2}+\left(\alpha^{4}-16 \pi \alpha^{2} \tilde{\gamma}\right)^{1 / 2}}{2}, \\
& r_{1}=\frac{\alpha^{2}-\left(\alpha^{4}-16 \pi \alpha^{2} \tilde{\gamma}\right)^{1 / 2}}{2} .
\end{aligned}
$$

Theorem 3: For this system, with

$$
\alpha^{2}>16 \pi \tilde{\gamma}>0
$$

one may pick $f$ satisfying

$$
0 \leqslant f(x, y) \leqslant \frac{c \exp \left(-\sqrt{r_{1}}|x-y|\right)}{|x-y|} .
$$
I.

We thus have two natural systems satisfying Condition

Before turning to a proof of Theorem 1 we first consider a simpler statistical mechanics model, a Gaussian distribution of continuous charges on a unit rectangular lattice.

$$
Z=\prod_{i}\left(\int d J_{i} e^{-\alpha / 2 J_{i}}\right) \exp \left[\left(\frac{-\beta}{2}\right) \sum_{i, j} J_{i}\left(\frac{\gamma}{r}+v\right) J_{j}\right] .
$$

There is a $J_{i}$ for each lattice site $i .1 / r$ denotes the Green's furction for a discrete Laplacian $\Delta$. With a notation similar to Eqs. (6)-(8) one has the pull-through formula (an integration by parts),

$$
I\left(J_{i} B\right)=\frac{1}{\alpha} I\left(\frac{\partial B}{\partial J_{i}}\right) .
$$

As in (5) one defines

$$
\phi=\sum\left(\frac{\gamma}{r}+v\right)_{i} J_{i}
$$

We want to study $\left\langle J_{y} \phi_{x}\right\rangle, y$ and $x$ lattice sites, and write

$$
\left\langle J_{y} \phi_{x}\right\rangle=\left\langle J_{y}\left\{\phi_{x}-\sum f_{i} J_{i}+\sum f_{i} J_{i}\right\}\right\rangle
$$

(the dependence of $f$ on $x$ is suppressed). We apply (27) to the third term in braces,

$$
=\frac{1}{\alpha} f_{y}+\left\langle J_{y}\left\{\phi_{x}-\sum f_{i} J_{i}-\frac{\beta}{\alpha} \sum f_{i}\left(\frac{\gamma}{r}+v\right)_{i j} J_{j}\right\}\right\rangle \text {. }
$$

If we can find an $f$ satisfying

$$
(\alpha+\beta \gamma(1 / r) *+\beta v *) f=\alpha\left(\frac{\gamma}{r}+v\right),
$$

an exact analogy of (10), we get

$$
\left\langle J_{y} \phi_{x}\right\rangle=\frac{1}{\alpha} f_{y},
$$

(31) and (32) directly yield

$$
\left\langle J_{i} J_{j}\right\rangle=\left(\frac{1}{\alpha+\beta \gamma / r+\beta v}\right)_{i j}
$$

Any correlation function can be calculated by this result and Wick's theorem for this Gaussian model. It follows that for such a Gaussian model if $\alpha, \beta, \gamma$ and $v$ are picked to ensure

$$
\left\langle J_{i} \phi_{j}\right\rangle \geqslant 0 \text { all } i \text { and } j,
$$

and (as is automatic)

$$
\left\langle\phi_{i} \phi_{i}\right\rangle \geqslant 0 \text { all } i,
$$

that it satisfies Conditions II, III.1, III.2, and III.3. It is easy to show that a Gaussian model approximating the models of Theorem 2 or Theorem 3, having the same $f$ in a discretized form, satisfies (34). That is, our Conditions II and III hold for the continuous approximations of our models, essentially the $\beta \rightarrow 0$ limit (with $\beta z$ fixed).

Returning to a proof of Theorem 1, we first exhibit an analog of the pull-through formula (27) in the classical statistical mechanics setting. We let $B$ be a functional of the $\left\{\rho_{i}(x)\right\}$. Then

$$
I\left(\rho_{i}(x) B\right)=z_{i} I\left(B^{(\cdot i x)}\right),
$$

where $B^{(\cdot i x)}$ is $B$ with $\rho_{i}(y)$ replaced by $\rho_{i}(y)+\delta(y-x)$. As in (29) we write

$$
\begin{aligned}
& \langle J(y) \phi(x)\rangle \\
& \quad=\left\langle J(y)\left\{\phi(x)-\int f(z) J(z)+\int f(z) J(z)\right\}\right\rangle
\end{aligned}
$$

and apply (36) to the last term in braces getting

$$
\langle J(y) \phi(x)\rangle=C+E+M,
$$

where $C$ will be a set of terms identically canceling, $M$ will be the main term, and $E$ will serve the place of an error. 


$$
\begin{aligned}
C= & =\left\langleJ ( y ) \int \left[\frac{\gamma}{r}+v-f-\sum z_{i} q_{i}^{2} \beta\right.\right. \\
& \left.\left.\times \int f\left(\frac{\gamma}{r}+v\right)\right] J\right\rangle .
\end{aligned}
$$

Applying the Laplacian to the coefficient of $J$ one gets exactly Eq. (10) in Condition I,

$$
M=\sum z_{i} q_{i}^{2} f(y, x)\left\langle e^{-\beta q, \phi(y)}\right\rangle .
$$

The expectations in (40) are all positive, their values determine the constant in (15) of Theorem 1.

$$
E=\sum z_{i} q_{i} \int f(z)\left\langle J(y)\left(e^{-\beta q_{i} \phi(z)}-1+\beta q_{i} \phi(z)\right)\right\rangle .
$$

By Condition III. 3 we assume $E \leqslant 0$. Thus from (38) we have

$$
0 \leqslant\langle J(y) \phi(x)\rangle=M+E \leqslant M,
$$

The first inequality is Condition II; this is Eq. (15). The statement of III. 1 trivially yields III. 3 for symmetric systems. The inequalities of (13) and (14) are identical by a simple application of (36).

Proceeding to collect some final points we note that it was only necessary to ensure $C \leqslant 0$, not that $C=0$, and this freedom may be helpful in some situations. It is amusing to attempt to eliminate the need for an error term in (38) by allowing the $f$ in (37) to be a functional of the $J$ 's. This was attempted in Ref. 4 with very limited success; other expressions like (38) were obtained with smaller error terms.

\section{ACKNOWLEDGMENT}

This work was supported in part by NSF Grant PHY 77-02187.

\section{APPENDIX}

We look at some properties of the Green's function for the discrete Helmholtz equation on a unit rectangular lattice,

$$
(-\Delta+a) g=4 \pi \delta \text {. }
$$

(I) In one dimension,

$$
g(x)=c^{\prime} e^{-k \mid x !},
$$

with

$$
e^{k}+e^{-k}-2=a .
$$

(II) In three dimensions $g\left(x_{1}, x_{2}, x_{3}\right)$, symmetric in its arguments, satisfies

$$
\sum_{x_{2}, x_{1}} g\left(x_{1}, x_{2}, x_{3}\right)=c^{\prime} e^{-k\left|x_{2}\right|},
$$

for the same $k$ as in (I).

(III) In three dimensions

$$
g \leqslant c^{\prime \prime} \exp \left(-\sum_{i} \frac{\left|x_{i}\right|}{2} \ln \frac{N_{i}+r_{i}}{N_{i}-r_{i}}\right),
$$

where

$$
r_{i}=\left|x_{i}\right| / \Sigma\left|x_{i}\right|
$$

and

$$
\frac{4\left(\Sigma N_{i}\right)^{2}+(6+a)^{2} r_{i}^{2}}{(6+a)^{2}}=N_{i}^{2}, \quad N_{i}>0 .
$$

(II) follows easily from (I). (I) is verified by directly substituting into the difference equation. (III) is derived from a random walk expression for the Green's function. ${ }^{5}$

'D. Brydges, P. Federbush, Commun. Math. Phys. 49, 233 (1976); Commun. Math. Phys, 53, 19 (1977).

D. Brydges, Commun. Math. Phys. 58, 313 (1978).

${ }^{3}$ D. Ruelle, Statistical Mechanics (Benjamin, New York, 1969)

${ }^{4}$ P. Federbush, "A Functional Relationship for the Two Point Correlation Function and Approximate Debye Screening in Classical Statistical Mechanics" (unpublished preprint).

'D. Brydges and P. Federbush, Commun. Math. Phys, 62, 79 (1978). 